Copyright (C) 2008 IEEE. Reprinted from:

L. E. Gurrieri, T. J. Willink, A. Petosa, and S. Noghanian, "Characterization of the Angle, Delay and Polarization of Multipath Signals for Indoor Environments," IEEE Transactions on Antennas and Propagation, vol. 56, no. 8, pp. 2710 - 2719, Aug. 2008. DOI: 10.1109/TAP.2008.927507, ISSN: 0018-926X.

This material is posted here with permission of the IEEE. Such permission of the IEEE does not in any way imply IEEE endorsement of any of the University of Ottawa's products or services. Internal or personal use of this material is permitted. However, permission to reprint/republish this material for advertising or promotional purposes or for creating new collective works for resale or redistribution must be obtained from the IEEE by writing to pubs-permissions@ieee.org.

By choosing to view this document, you agree to all provisions of the copyright laws protecting it. 


\title{
Characterization of the Angle, Delay and Polarization of Multipath Signals for Indoor Environments
}

\author{
Luis E. Gurrieri, Tricia J. Willink, Senior Member, IEEE, Aldo Petosa, Senior Member, IEEE, and \\ Sima Noghanian, Senior Member, IEEE
}

\begin{abstract}
A high-resolution channel sounding technique has been used to investigate the cross-polarization of electromagnetic waves in the 5-6 GHz band. Experiments were performed in two non-line-of-sight indoor locations, and it was found that there is a strong dependency of the cross-polarization of multipath components on the elevation angle-of-arrival. For a vertically polarized transmitting antenna, clusters of co-polarized multipath components were confined predominantly to the region around the horizontal plane that contains the virtual line-of-sight between transmitter and receiver. In contrast, cross-polarized signals were detected for a variety of elevation angles with considerable power levels. The surroundings of the receiver were identified as the principal source of depolarized signals. In addition, time dispersion analysis of the multipath signals led to the determination of the AoAs where there is a strong correspondence between co- and cross-polarized signals as a consequence of the partial depolarization of MPCs. This work supports the exploitation of the joint space and polarization diversities in indoor propagation scenarios to improve the system performance.
\end{abstract}

Index Terms-Electric field measurement, indoor propagation, multipath channels, polarization.

\section{INTRODUCTION}

$\mathbf{T}$ HE increasing utilization of wireless links in indoor scenarios and the limited available bandwidth demand innovative solutions to increase spectral efficiency. The use of joint space and polarization diversities has been proposed as an attractive alternative to boost the system capacity.

Lee and Yeh [1] pioneered the study of polarization diversity for mobile communications using a combination of half-wavelength dipoles and loop antennas in the mobile station (MS) and two orthogonally polarized pyramidal horns at the base station (BS). By transmitting and receiving both orthogonally polarized signals, they studied the amount of diversity gain expected in urban mobile environments. Kozono et al. [2] measured orthogonally polarized signals as a function of the distance between MS and BS. Cox et al. [3] extended the study of depolarized

Manuscript received March 23, 2007; February 15, 2008. Published August 6, 2008 (projected). This work was supported in part by Defence Research and Development of Canada (DRDC), National Science and Engineering Research Canada (NSERC) and in part by the University of Manitoba.

L. E. Gurrieri is with the Communications Research Centre, Ottawa, ON K2H 8S2, Canada and also with the Department of Electrical and Computer Engineering, Queen's University, Kingston, ON K7L 3N6, Canada (e-mail: luis. gurrieri@crc.ca).

T. J. Willink and A. Petosa are with the Communications Research Centre, Ottawa, ON K2H 8S2, Canada.

S. Noghanian is with the Department of Electrical and Computer Engineering, University of Manitoba, Manitoba R3T 2N2, Canada.

Digital Object Identifier 10.1109/TAP.2008.927507 signals to the case of propagation within and around houses and large buildings. The authors showed that depolarized signals were distributed uniformly around the receiver except in a few residential locations. Furthermore, high decoupling levels were reported when transmitting from inside houses and even higher decoupling was found within large buildings. Vaughan studied the polarization diversity gain that arises when transmitting vertically polarized signals from the MS and receiving in both polarizations at the BS [4]. Higher polarization diversity gain was reported for urban scenarios where scattering and reflections are more significant than in the suburban case. All of these early measurement campaigns were made in the UHF band. Only outdoor and indoor-outdoor propagation scenarios were considered for the performance assessment of polarization diversity systems; the angle-of-arrival (AoA) of both polarization signals were not reported.

In recent years, more sophisticated measurements campaigns have been made, including the joint spatiotemporal distribution of multipath components (MPCs). In addition, polarization diversity was revisited in [5], motivated by spectral efficiency concerns and the data rates envisioned by the next generation of wireless communication systems [6], [7]. One of the first joint spatiotemporal models for indoor propagation was made by Wallace et al. [8] who successfully extended the Saleh-Valenzuela model to include the azimuth AoA in [9]. Only the co-polarized MPCs in the horizontal plane that contains the radiation center of the transmitter and receiver antennas are included in this model. In [10], Kallioka et al. presented a sounding method to characterize the propagation channel in terms of azimuth and elevation AoAs which was used to measure the depolarization and spatial power distribution in picocell environments at $2.15 \mathrm{GHz}$ [11]. Recently, the use of virtual arrays in combination with the space alternating generalized expectation-maximization (SAGE) algorithm has become an increasingly popular method to determine the angle-of-departure (AoD), AoA, complex weight, delay, Doppler frequency and polarization of MPCs [12], [13]. Using this technique, Chong et al. [14] characterized the multipath cluster statistics in SIMO indoor channels at $5.2 \mathrm{GHz}$. Their study focused on co-polarized MPC clusters in the AoA/delay domain; only the azimuth AoA in the horizontal plane was considered. Extending this research to MIMO channels for indoor scenarios, Fleury et al. reported the formation of clusters of co-polarized MPCs as a function of the AoD and AoA in [15].

The joint spatiotemporal characterization of co- and cross-polarized multipath signals in indoor environments is a particularly challenging problem. Indoor channels vary from building to 
building and among locations within the same building. Peculiarities of the indoor scenario such as the location and size of scatterers determine the formation of depolarized MPCs [16], [17]. The characterization of the static indoor channel in the 5.1-5.75 $\mathrm{GHz}$ frequency band for both received polarizations and for vertically polarized, omnidirectional transmitter antennas is reported herein. The spatial and temporal distributions of MPCs and the impact of depolarized signals in the total received power has been studied for typical non-line-of-sight (NLOS) confined environments where the number of interactions between the signal and the indoor clutter is high.

The channel sounding and data postprocessing technique are described in Section II. A description of location of the experiments is followed by the received power and delay characterizations as a function of the AoA and polarization in Section III. Conclusions from this experiment are drawn in Section IV.

\section{Channel Measurement AND AnAlysis}

The indoor channel measurement campaign that was undertaken at the Communication Research Centre (CRC) in Ottawa is described in this section. The complex channel amplitude, $\beta$, or channel frequency response (CFR), was measured in a frequency band of relevance for indoor wireless communications such as those specified in the HIPERLAN/2 and IEEE802.11a standards. To measure the indoor channel with sufficient accuracy, the sounder MPC resolution was required to be $20 \mathrm{~ns}$ or better to enable the detection of MPCs with path length differences of $60 \mathrm{~cm}$, which is the average distance between scatterers in an indoor cluttered scenario. In addition, an AoA resolution of at least $10^{\circ}$ is required, based on recent reports of indoor MPC cluster angular spreads [14], [15]. Furthermore, these specifications apply to the channel characterization in both polarizations.

A sequential mechanical scanning was used to measure the CFR for selected azimuth and elevation AoAs as described in Section II-A. The characteristics of the sounder transmitter and receiver antennas are presented in Sections II-B and C. The acquired channel measurements were post-processed to reduce the distortive effects introduced by the receiver antenna response, as presented in Section II-D. The procedure used to obtain the final complex impulse response (CIR) in AoA and delay domains from the CFR and the determination of the MPC threshold is also described in Section II-D.

\section{A. The Channel Sounder}

The acquisition apparatus consists of a narrow-beam, wideband antenna mounted on a positioning platform specially designed to mechanically scan the radio environment for specific azimuth $(\phi)$ and elevation $(\theta)$ angles in both polarizations. The measurements were made using a network analyzer (NA) which generated a continuous wave (CW) swept across the 5.10-5.85 $\mathrm{GHz}$ band in $1.875 \mathrm{MHz}$ frequency steps; this sampling bandwidth provides a MPC delay resolution of less than $1.3 \mathrm{~ns}$ before the signal is windowed to reduce the temporal ripples introduced by the scanning window. At each scanning angle, the complex channel amplitude was sampled at the frequencies of interest. The sounding $\mathrm{CW}$ was transmitted from the two selected indoor locations using a biconical, vertically polarized antenna. A computer was used to control the platform and NA

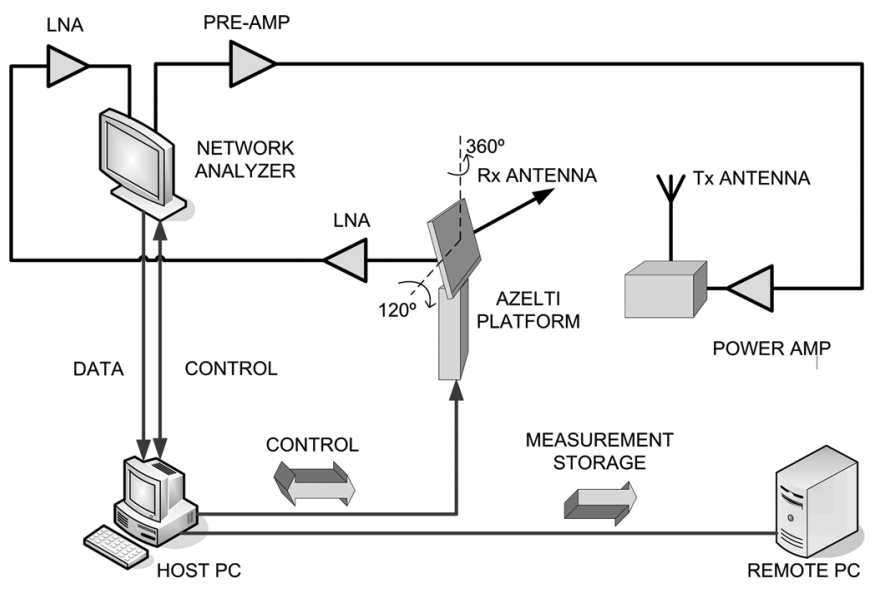

Fig. 1. Measurement setup based on the Azimuth-Elevation-Time (AZELTI) channel sounder platform.

remotely using the existing wired LAN of the building, thereby eliminating the need for personnel in the surroundings of the test area. The channel sounding block diagram is shown in Fig. 1.

The received signal at frequency $\gamma$ for azimuth $\phi$ and elevation $\theta$ is given by

$$
\begin{aligned}
H_{i}(\phi, \theta, \gamma)=\sum_{n=1}^{N_{\phi}} \sum_{m=1}^{N_{\theta}} \sum_{k=1}^{N_{\gamma}} \beta_{i}(n, m, k) \delta\left(\phi-\phi_{m}\right) \\
\times \delta\left(\theta-\theta_{n}\right) \delta\left(\gamma-\gamma_{k}\right)
\end{aligned}
$$

where $\delta$ is the Dirac's delta function; the index $i, i \in\{V, H\}$, is used to denote vertical or horizontal polarization, respectively; $N_{\phi}, N_{\theta}$, and $N_{\gamma}$ are the total number of resolution bins in azimuth, elevation and frequency, respectively; $\phi_{m}$ and $\theta_{n}$ specify the look angle; $\gamma_{k}$ is the $k$ th frequency sample; and the term $\beta_{i}(n, m, k)$ represents the complex channel amplitude at the specified angle and frequency indices. Five consecutive measurements obtained at each position of the platform were averaged to give $\beta_{i}(n, m, k)$ at each of the $N_{\phi}=72, N_{\theta}=36$ positions and $N_{\gamma}=400$ frequencies.

In the system of coordinates used, the vertical axis, $\theta=0^{\circ}$, corresponds to the direction perpendicular to the floor plane pointing to the ceiling; and $\theta=90^{\circ}$ is on the horizontal plane containing the radiation centers of transmitter and receiver antennas. The direction $\phi=0^{\circ}$ corresponds to the look angle from the transmitter to the receiver for any location. The CFR was sampled for AoAs between $0^{\circ}$ and $355^{\circ}$ in azimuth and between $30^{\circ}$ and $150^{\circ}$ in elevation in steps of $5^{\circ}$ in both dimensions. A total of five snapshots of the CFR for each AoA were averaged to minimize the noise effects. The frequency response of the attenuators and amplifiers were considered in the signal post-processing.

\section{B. The Transmitter Antenna}

The objective of the experiment was to measure the depolarizing effects of the propagation environment, which required a transmitter antenna with good cross-polarization discrimination (XPD) characteristics. The transmitter antenna used was a biconical, vertically polarized antenna, designed and built at the Communications Research Centre. 


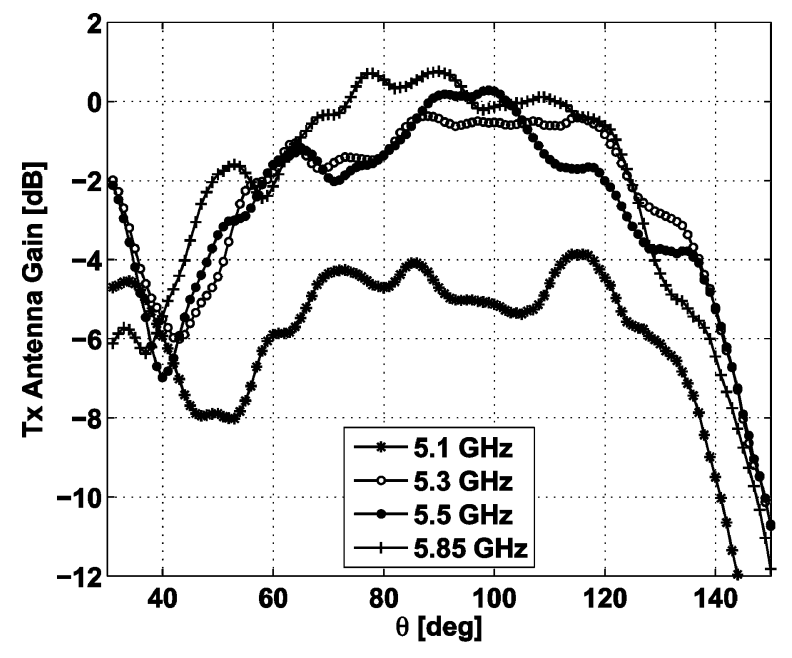

(a) Transmit antenna co-polarized response

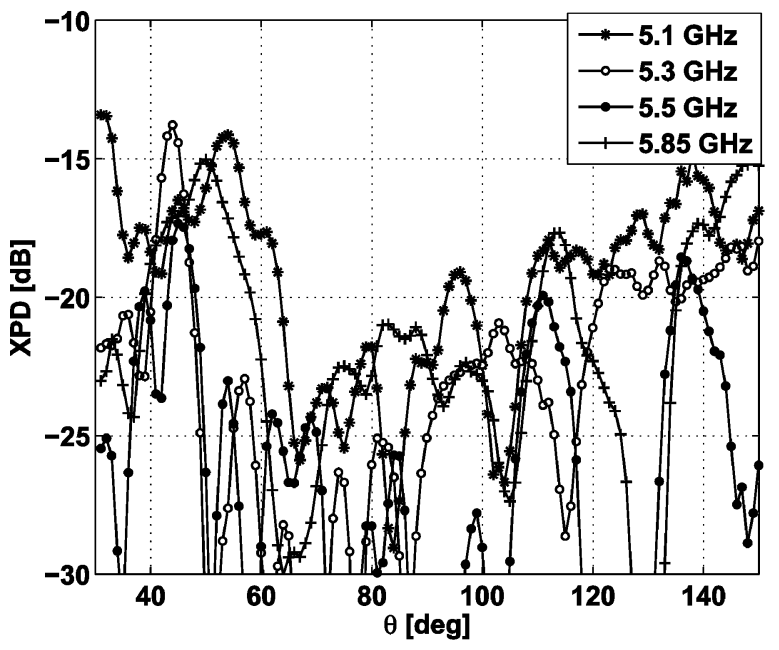

(b) Cross-Polarization discrimination

Fig. 2. Transmitter antenna characteristics as a function of the elevation angle for different frequencies.

The transmitter antenna was characterized in an anechoic chamber. The transmitted power and XPD over the operative frequency band are shown in Fig. 2. The XPD was better than $18 \mathrm{~dB}$ over the range of frequencies and a wide range of elevation angles. Except at the extreme low end of the frequency range, the XPD was below $21 \mathrm{~dB}$ for elevation angles between $70^{\circ}$ and $105^{\circ}$.

\section{The Receiver Antenna}

The performance of the indoor channel sounder is dependent on the receiver antenna characteristics. Scatterers located in the near-field of the antenna may cause erroneous measurements. In addition, the sounding system antenna characteristics such as bandwidth, half-power beam-width (HPBW) and side-lobe levels (SLLs) determine the delay/AoA resolution. For these experiments, the antenna bandwidth must be large enough to guarantee the desired multipath delay resolution and the HPBW needs to be narrow enough to meet the required AoA resolution.

The receiver antenna used in this campaign was a square planar array of 8 by 8 elements built using a stacked patch configuration. The antenna bandwidth was $1.1 \mathrm{GHz}(4.9-6.0 \mathrm{GHz})$

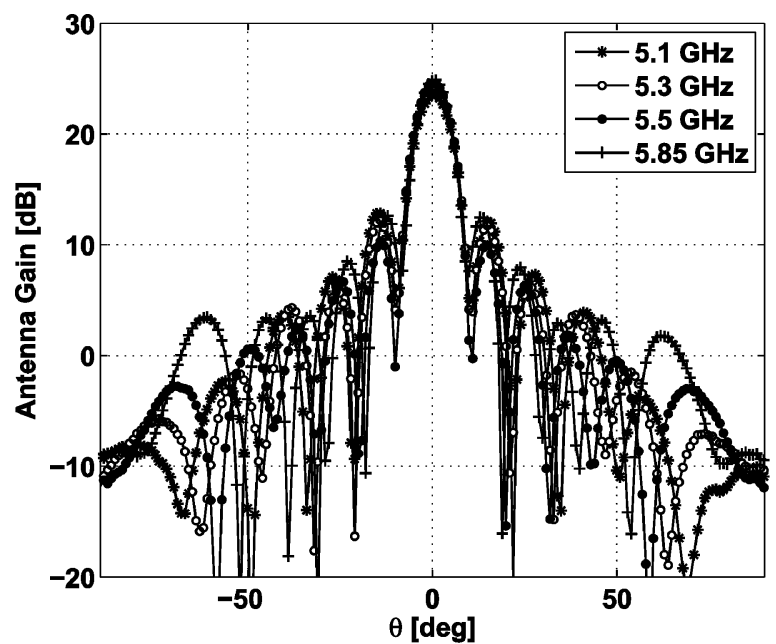

(a) Vertical Plane

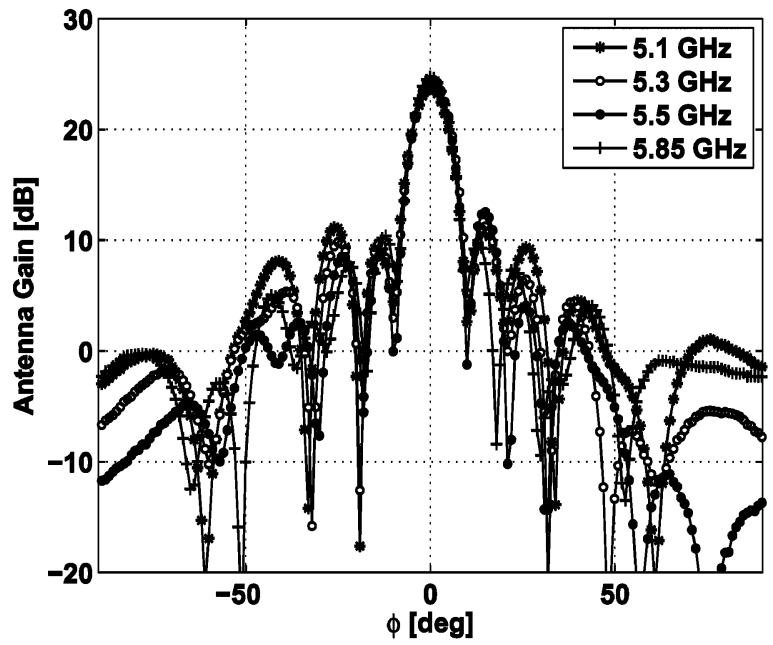

(b) Horizontal Plane

Fig. 3. Receiver antenna co-polarized radiation patterns for different frequencies.

and its HPBW was consistently less than $10^{\circ}$ in its frequency of operation. It was characterized in an anechoic chamber before the measurement campaign took place. Fig. 3 shows the radiation patterns in both principal planes for selected frequencies.

The cross-polarization discrimination (XPD) was characterized with the antenna response in an anechoic chamber before the experiment took place. As shown in Fig. 4, the XPD was better than $26 \mathrm{~dB}$ within the relevant directions. Observe that a $23 \mathrm{~dB}$ or better discrimination was measured at the extreme of the operative frequency band and only for certain angles in the azimuthal plane. A discrimination of the same order is observed in the elevation response which is reduced to $20 \mathrm{~dB}$ at the extreme of the operative band. To measure depolarized MPCs, the mechanical scanning was repeated after rotating the receiver antenna $90^{\circ}$ around its geometric center. The use of the same antenna radiation pattern to characterize both polarizations enabled an accurate comparison between co- and cross-polarized measurements.

The minimum allowable distance to obstacles around the sounding platform was estimated based on the antenna nearand far-field approximated ranges. Near-field patterns estimated using the XCcel simulation tool [18] for 50 and $100 \mathrm{~cm}$ 


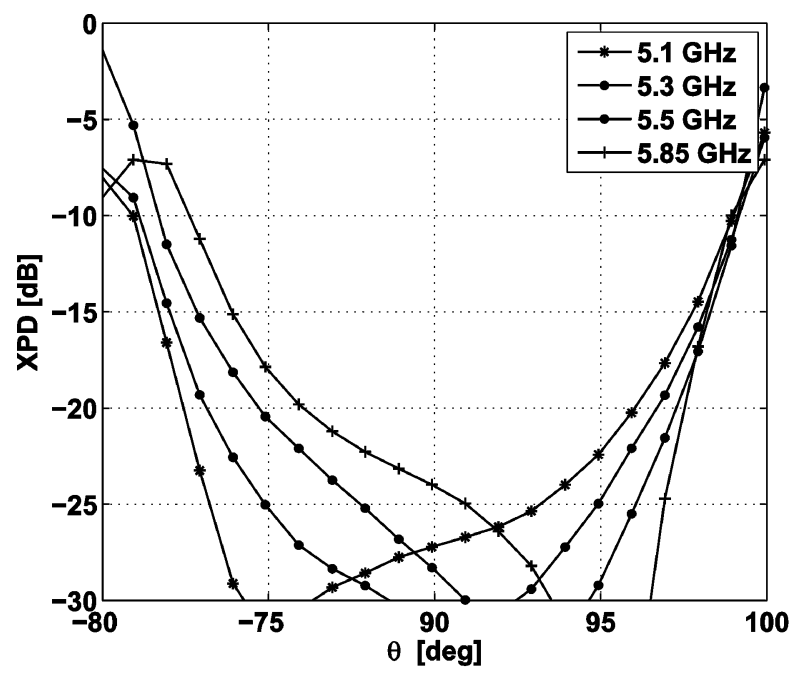

(a) Vertical Plane

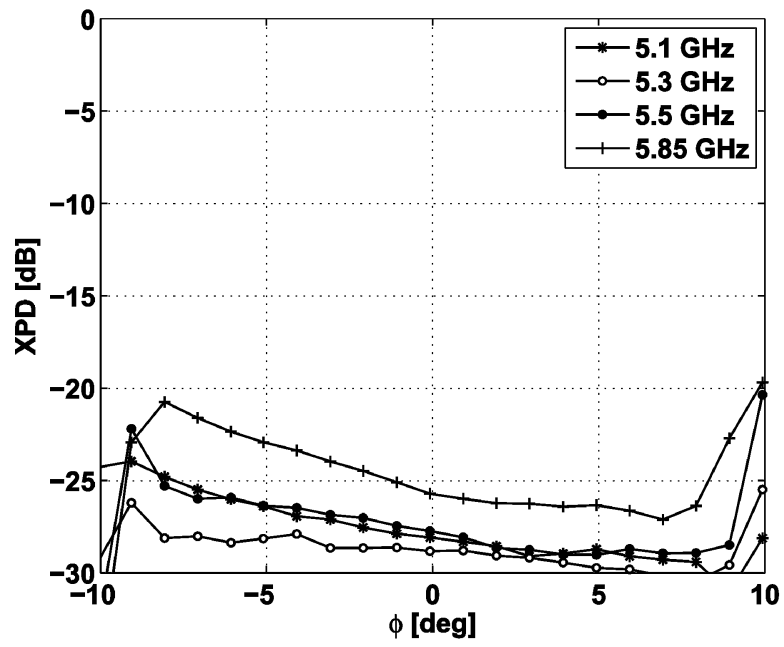

(b) Horizontal Plane

Fig. 4. Cross-polarization discrimination of the receiver antenna for different frequencies.

are contrasted against the far-field measured pattern in Fig. 5. From these results, a fast convergence to the far-field was expected beyond $100 \mathrm{~cm}$. For additional assurance, the sounding system was located such that the nearest obstacles were more than $175 \mathrm{~cm}$ from the antenna center. Finally, the elevation scanning range was limited to $30^{\circ}-150^{\circ}$ to avoid errors due to the proximity of floors and ceilings.

\section{Data Post-Processing}

The CFR snapshots were acquired with a sampling rate of $5^{\circ}$ in azimuth and elevation, which is half the antenna main beamwidth. This spatial oversampling reduces aliasing of MPCs in the spatial domain, and thereby provides an improved resolution in the data post-processing using the CLEAN algorithm.

The error in the identification of MPCs due to the interaction between main-lobe and side-lobes of the antenna radiation pattern was reduced using the CLEAN algorithm which is an iterative deconvolution procedure originally used for debluring of astronomical images [19]. The antenna response for each sample

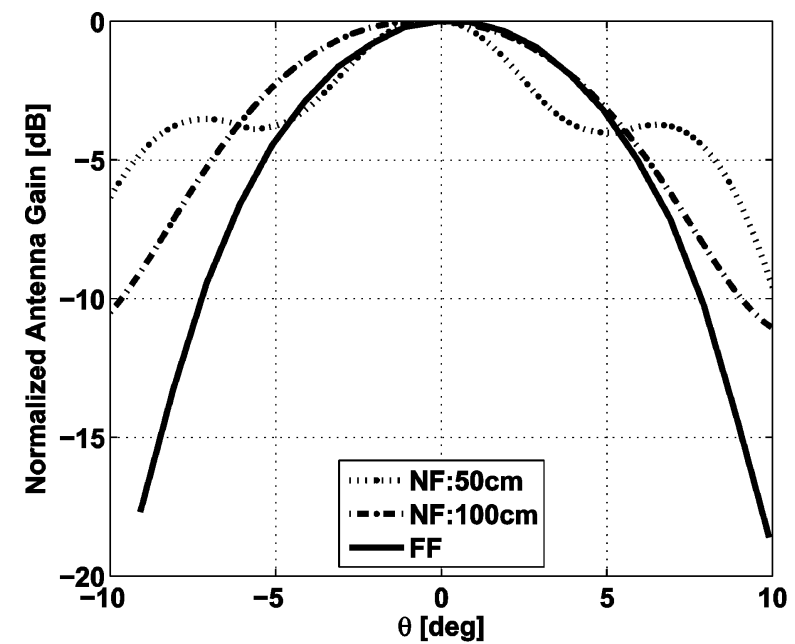

Fig. 5. Near-field (NF) and far-field (FF) patterns in the vertical plane at $5.2 \mathrm{GHz}$.

frequency in both principal planes is required to apply the 2-D implementation of the CLEAN algorithm [20]. Since the antenna response was measured every $100 \mathrm{MHz}$ in the band of interest, intermediate responses were obtained by linear interpolation using the available data.

The resolution of the system was obtained by simulating the reception of two or more MPCs and using the antenna radiation pattern under the same noise level. The sample distance (AoA) between MPCs and their relative power levels were independently controlled in the simulation. As a result, MPCs with a minimum separation of $5^{\circ}$ in azimuth and elevation were successfully identified using the post-processing method used herein.

A three-term Blackman-Harris window was applied to the CFR to reduce the temporal sidelobes introduced by the rectangular frequency window [21]. The CFRs at a given look angle $(\theta, \phi)$, which are the complex channel amplitudes in the discrete frequency domain, were inverse Fourier transformed to yield the CIR in the time domain for that AoA.

Finally, the constant false alarm rate (CFAR) method [22] was used to obtain the model order and separate the valid MPCs from the background noise. The first step was to estimate the noise amplitude, which was assumed to be additive white Gaussian, based on the measured samples. The amplitude of this noise is Rayleigh distributed with noise power $\sigma_{N}^{2}$. Therefore, the probability that a sample amplitude exceeds a defined threshold $\alpha$ is given by

$$
P=\exp \left(-\frac{\alpha^{2}}{2 \sigma_{N}^{2}}\right) .
$$

The noise threshold was estimated making $\alpha$ equal to the median amplitude $\sigma_{m}$ calculated over all samples on each location and making the probability of exceeding this amplitude 0.5 . Therefore, the estimated noise amplitude is given by $\sigma_{N} \simeq 0.85 \cdot \sigma_{m}$. The MPC threshold was obtained by defining a new amplitude $\alpha=\vartheta \cdot \sigma_{N}$ so that the probability of false alarm $P_{f}$ is given by

$$
P_{f}=\exp \left(-\frac{\vartheta^{2}}{2}\right)
$$




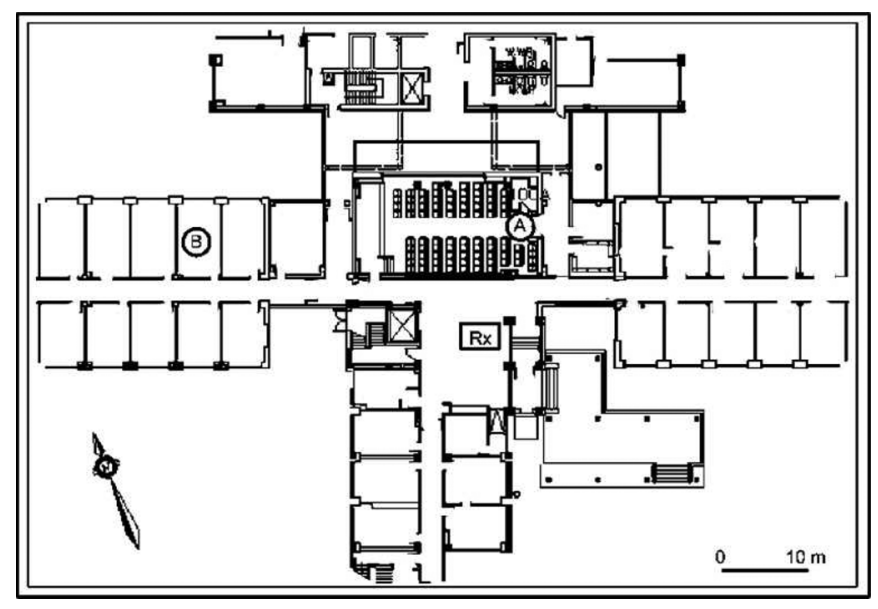

Fig. 6. Transmitter locations $\mathrm{A}$ and $\mathrm{B}$, and receiver location $\mathrm{Rx}$.

The false detection tolerance was set to 1 in $10^{7}$ samples, or $P_{f}=10^{-7}$, and, obtaining $\vartheta$ from (3), the MPC threshold was calculated at approximately $15 \mathrm{~dB}$ above the noise level.

\section{Channel Characterization}

The results of the characterization of indoor channel in terms of the spatial distribution and delay spread of the MPCs for both polarizations are presented in this section. First, a brief description of the two transmitter scenarios used in this experiment is provided in Section III-A to illustrate the different propagation mechanisms that may explain the results. Then, the MPC spatial distribution at the receiver is presented in Section III-B followed by the MPC delay spread analysis in Section III-C.

\section{A. Location of the Experiments}

The two NLOS locations chosen for this experiment are shown in Fig. 6. In propagation scenario A, the transmitter was located in an auditorium with many potential scatterers. These sources of MPCs, e.g., metallic folded chairs and ventilation ducts, could create depolarized signals departing from different angles near the transmitter. On the other hand, in propagation scenario $\mathrm{B}$, the transmitter was located in a fully furnished office; in this case, there is a single dominant transmission path along the corridor, hence it is expected that the objects in the receiver neighbourhood will have more impact than those in the transmitter area. For both scenarios, heating ducts, pipes and electric wires run above the ceiling. Double layer plywood, concrete and brick walls, steel reinforced concrete columns as well as typical office equipment were present. In order to have maximum control over the variables that could affect the channel measurement, the experiments were conducted during the weekend when the number of people in the area was minimal.

Considering the shortest distance between transmitter and receiver, the approximate elevation angles for a single bounce are $72^{\circ}, 99^{\circ}$ in Location A and $82^{\circ}, 96^{\circ}$ in Location B. For double bounces (floor/ceiling), the elevation angles are $90 \pm 26^{\circ}$ and $90 \pm 14^{\circ}$ in Locations A and B, respectively.

\section{B. The Received Power Characterization}

One characteristic found at both transmitter locations is that co-polarized MPCs are mainly located in the horizontal plane. The relatively large margin between co- and cross-polarized signals arriving from this direction $\left(\theta=90^{\circ}\right)$ justifies taking into account only the co-polarized signals when using omnidirectional, vertically polarized antennas [23]. As the experiments reported herein provided adequate delay and angular MPC resolution, it was possible to measure significant depolarized power coming from AoAs outside the horizontal plane for both transmitter locations. This effect can be attributed to rotations in the electric field of the electromagnetic (EM) waves due to reflections from the floor and ceilings, which create depolarized MPCs in the immediate surroundings of the receiver.

In order to compare the results for both polarizations, the total power coming from distinctive AoAs was normalized as follows:

$$
P_{i}(\phi, \theta)=\frac{1}{P^{\max }} \sum_{k=1}^{N_{\tau}}\left|h_{i}\left(\phi, \theta, \tau_{k}\right)\right|^{2}
$$

where: $h_{i}\left(\phi, \theta, \tau_{k}\right)$ is the CIR as a function of the azimuth $(\phi)$, elevation $(\theta)$ and the delay $\left(\tau_{k}\right) ; N_{\tau}$ is the number of resolution bins in delay; the index $i$ denotes the polarization state, $\mathrm{V}$ or $\mathrm{H}$; and $P^{\max }$ represents the maximum MPC power for any AoA and polarization for a given scenario.

In Fig. 7, the received power due to vertical (co-polarized) and horizontal (cross-polarized) signals is shown for a subset of the AoAs in scenario A. Note that the direction of reference $\phi=0^{\circ}$ and $\theta=90^{\circ}$ is defined as the direction from the receiver to the transmitter for each location according to Fig. 6 with the positive azimuth angles corresponding to the counter-clockwise direction of scanning.

The indoor clutter surrounding the transmitter in scenario A, as described in Section III-A, creates signals departing from diverse elevation angles by multiple reflections. As a result of these interactions, a large number of MPC signals depart from the neighbourhood of the transmitter location in scenario A already depolarized. However, most of these signals suffer attenuation beyond the detection level before arriving at the receiver. Therefore, the stronger MPCs that arrive at the receiver come from AoAs in the virtual line-of-sight (LOS) between transmitter and receiver, i.e., between $0^{\circ}<\phi<20^{\circ}$ and $80^{\circ}<\theta<110^{\circ}$. These signals suffered a single interaction with the environment, in this case transmission through the hall wall.

Fig. 8 shows the cross-polarization ratio (XPOL) for scenario A, which was used to compare the amount of depolarization for different AoAs. The XPOL is defined as follows:

$$
\operatorname{XPOL}(\phi, \theta)=\frac{P_{H}(\phi, \theta)}{P_{V}(\phi, \theta)}
$$

where $P_{V}$ and $P_{H}$ are the total power received due to vertically and horizontally polarized signals, respectively, as a function of both azimuth and elevation angles. The maximum depolarization is localized in two main MPC clusters around $0^{\circ}<\phi<$ $30^{\circ}$ and $90^{\circ}<\phi<100^{\circ}$ in azimuth and for $\theta>90^{\circ}$. The 


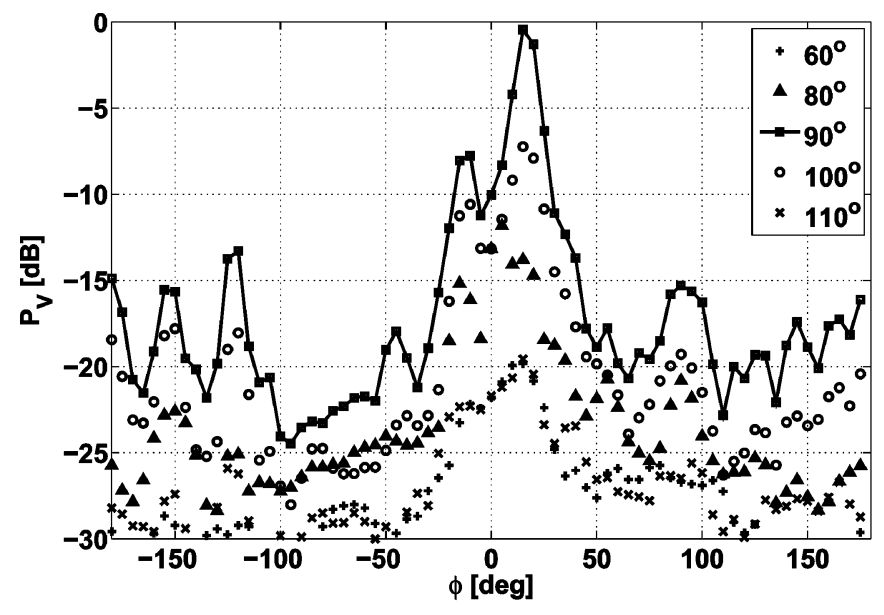

(a) Co-polarized component

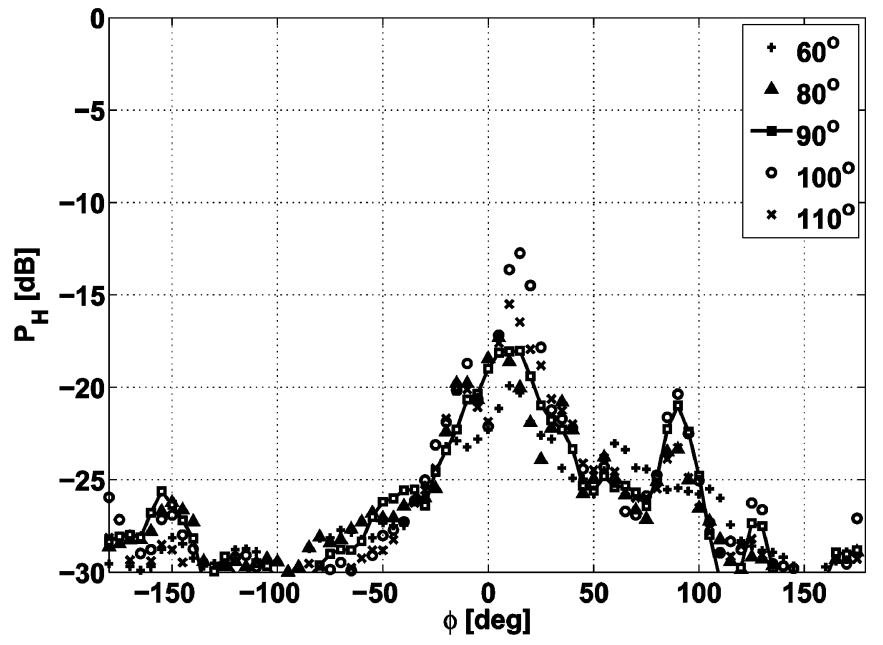

(b) Cross-polarized component

Fig. 7. Normalized received power due to co- and cross-polarized MPCs in scenario A.

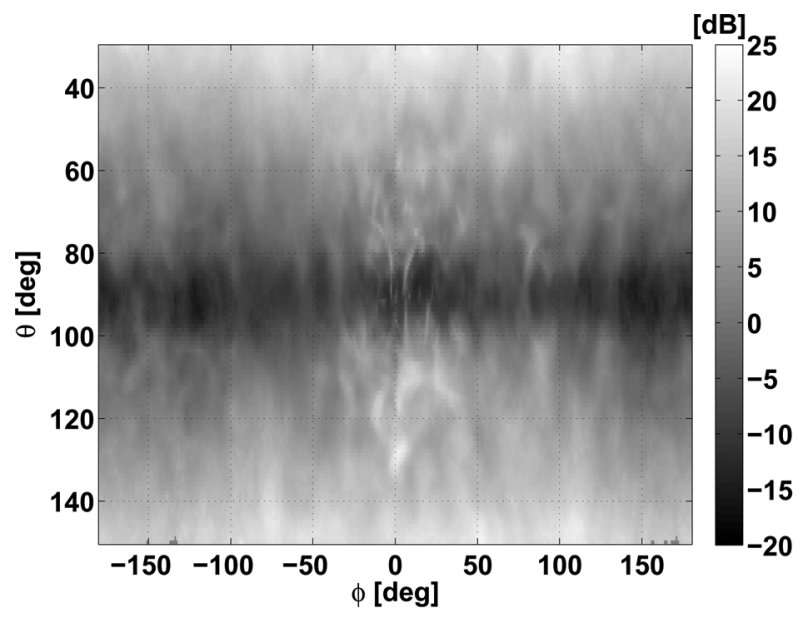

Fig. 8. XPOL distribution for scenario A.

first of these MPC clusters is a result of signals that are transmitted through the wall and have enough power to be reflected off the floors and ceilings, generating partially and totally depolarized MPCs. The maximum received cross-polarized power

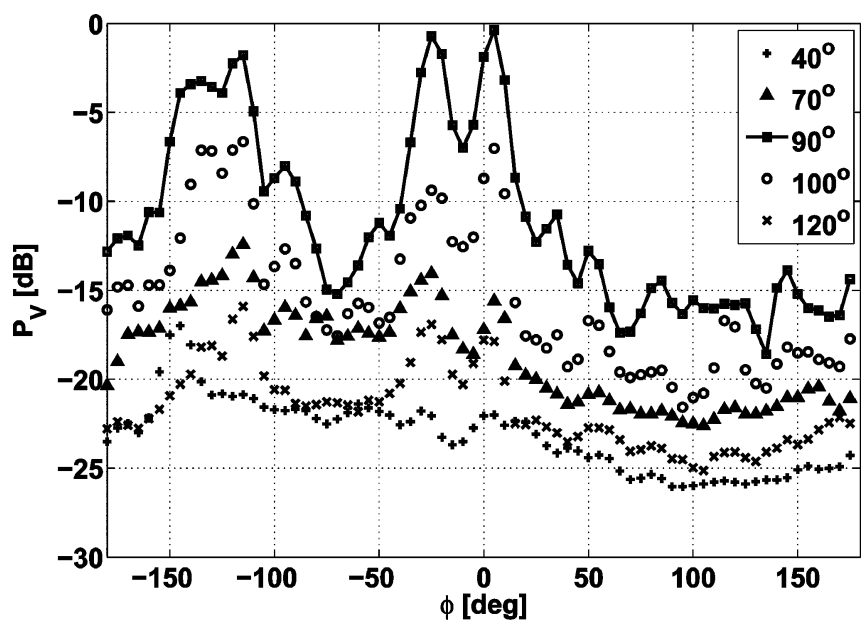

(a) Co-polarized component

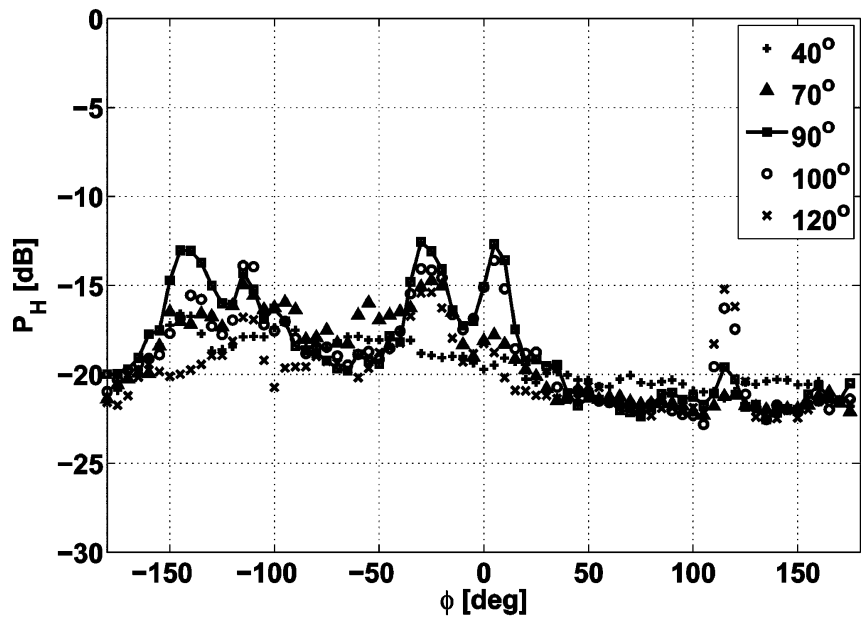

(b) Cross-polarized component

Fig. 9. Normalized received power due to co- and cross-polarized MPCs in scenario $\mathrm{B}$.

level is about $-12 \mathrm{~dB}$ below the co-polarized level at $\phi=10^{\circ}$. The second largest peak in the XPOL $\left(90^{\circ}<\phi<100^{\circ}\right)$ can be attributed to reflections from the metallic door of the elevator. This effect makes evident the importance of the physical nature of indoor clutter in the immediate surroundings of the receiver in the formation of depolarized MPCs. Note that for low and high elevation angles the power level due to cross-polarized MPCs surpasses the co-polarized level.

The depolarization of MPCs for the transmitter location in scenario B exhibits even more spread in elevation than in location A, as shown in Fig. 9. Note that for this scenario the azimuth angle $\phi=0^{\circ}$ was shifted $78^{\circ}$ with respect to the azimuth reference used in scenario A to represent the new virtual LOS. Two clusters located at $\phi=120^{\circ}$ in azimuth and $\theta=110^{\circ}$ and $\theta=120^{\circ}$ in elevation, respectively, as shown in Fig. 10, are originated by reflections from a metal room divider behind the wall in that direction. This is consistent with similar observations reported in [24]. While the first power cluster arriving from $\theta=100^{\circ}$ can be associated with MPCs with coand cross-polarized components as shown in Fig. 9(a) and (b), respectively, the signal detected from $\theta=120^{\circ}$ is due to predominantly cross-polarized MPCs. 


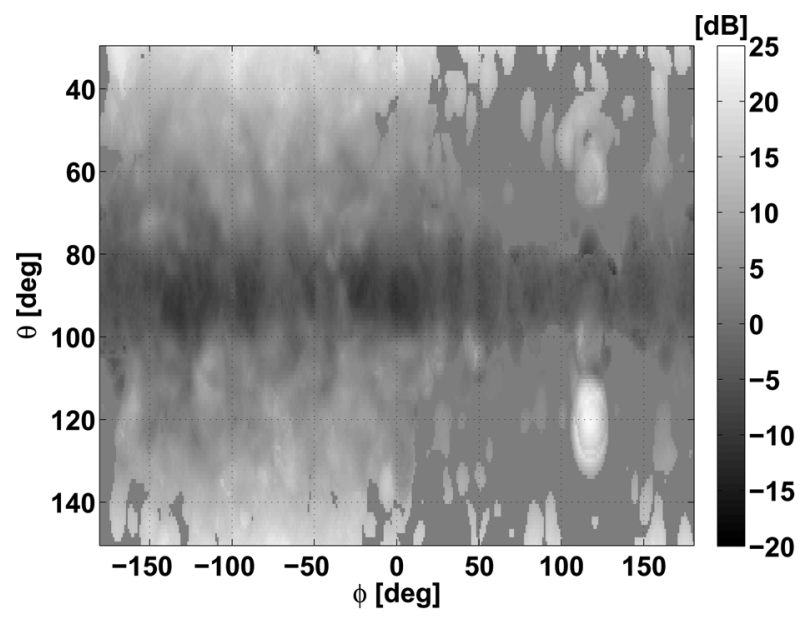

Fig. 10. XPOL distribution for scenario B.

The topology of the propagation scenario B is comparable to a dielectric canyon, which acts as a lossy waveguide favouring the propagation of vertically polarized components. Horizontally polarized MPCs caused by interactions in the transmitter surroundings do not have enough power to be detected at the receiver. For this transmitter location, co-polarized signals are stronger for the virtual LOS at $-40^{\circ}<\phi<10^{\circ}$ and from back reflections of signals coming from the direction $-160^{\circ}<\phi<$ $-100^{\circ}$, as can be seen in Fig. 9(a). In contrast with scenario A where the depolarized signal is enhanced for particular AoAs, the depolarized signals from scenario B are more uniformly spread in azimuth around the receiver as seen in Fig. 9(b). Another distinctive effect in this scenario is that depolarized signals are 10-fold stronger than those in scenario A for AoAs in the direction of floors and ceilings. The XPOL measured can be attributed to the decoupling of vertical components arriving at the receiver from the horizontal plane after one or more interactions with the walls. The maximum depolarization was symmetrically located in two clusters above and below the horizontal plane around $\phi=120^{\circ}$, as can be seen in Fig. 10. Oblique reflections from two metal panels (elevator doors) perpendicularly oriented with respect to the wall explain the maximum XPOL concentration observed for that azimuth angle.

The depolarization spatial distribution shows that the obstacles in the receiver surroundings are the main source of depolarization [25]. The indoor environment favours the propagation of vertically polarized signals, which retain enough power to be depolarized after being reflected off obstacles in the receiver proximity. Depolarized signals created in the transmitter proximity by the same mechanism are attenuated too much to be detected. Note that a large XPOL does not necessarily imply stronger horizontally polarized MPCs, but rather, may indicate weaker or no vertically polarized MPCs.

\section{Multipath Signal Delay Dispersion}

The data rate supportable in a MPC environment is dependent on the signal temporal dispersion. The root-mean-squared (rms) delay spread, denoted by $\sigma_{\text {rms }}$, is a measure of this dispersion and was used to characterize the channel in both polarizations.

Early characterizations in similar indoor environments reported significant differences in terms of $\sigma_{\text {rms }}$ when directive antennas are used at the receiver side [26]. The antenna radiation pattern affects $\sigma_{\text {rms }}$ directly by changing the number of MPCs in the received signal. In these experiments, the high delay resolution of the channel sounding technique enabled the detection of a large number of MPC clusters in the time-domain. The use of wider HPBW antennas and lower temporal resolution would cause MPCs to combine non-coherently, leading to different $\sigma_{\text {rms }}$ estimates [17].

The delay dispersion as a function of the AoA, $\sigma_{\mathrm{rms}}$, is obtained from [27]

$$
\sigma_{\mathrm{rms}}(\phi, \theta)=\sqrt{\frac{\sum_{k=1}^{N}\left(\tau_{k}^{(\phi, \theta)}-\overline{\tau_{\phi, \theta}}\right)^{2}\left|h\left(\phi, \theta, \tau_{k}^{(\phi, \theta)}\right)\right|^{2}}{\sum_{k=1}^{N}\left|h\left(\phi, \theta, \tau_{k}^{(\phi, \theta)}\right)\right|^{2}}}
$$

where

$$
\overline{\tau_{\phi, \theta}}=\frac{\sum_{k=1}^{N}\left(\tau_{k}^{(\phi, \theta)}-\tau_{o}^{(\phi, \theta)}\right)\left|h\left(\phi, \theta, \tau_{k}^{(\phi, \theta)}\right)\right|^{2}}{\sum_{k=1}^{N}\left|h\left(\phi, \theta, \tau_{k}^{(\phi, \theta)}\right)\right|^{2}}
$$

and $\tau_{k}^{(\phi, \theta)}$ is the ToA of the $k$ th MPC relative to the arrival time of the first valid MPC, $\tau_{o}$, in all cases for a given AoA, $(\phi, \theta)$.

Fig. 11 shows the power distribution as a function of both azimuth angle and delay for scenario $\mathrm{B}$ in the horizontal plane $\left(\theta=90^{\circ}\right)$. This direction was chosen since most of the power is coming from this elevation angle. Most of the co-polarized MPC clusters in Fig. 11(a) can be directly associated with depolarized components of the co-polarized signal in Fig. 11(b). Note that the power level for both polarizations was normalized as discussed in Section III-B.

Co- and cross-polarized signals exhibit similar time dispersion for AoAs where the co-polarized power is dominant. For scenario A, for example, at the same AoA where the co-polarized power is dominant, the $\sigma_{\text {rms }}$ values measured for both orthogonally polarized signals were similar as can be seen by comparing Figs. 7(a) and 12(a). The same effect was observed for scenario B, as can be seen from Figs. 9(a) and 12(b). This observation is due to the MPCs being only partially depolarized.

\section{Sources of Error}

If the signal energy is considered to be solely vertically polarized, the error sources contributing to the co-polarized measurement are: (a) depolarization at the receiver antenna of the component that was cross-polarized by the propagation environment between the transmitter and receiver; and (b) depolarization at the transmitter antenna, where the component is subsequently cross-polarized by the propagation environment. The XPD of the two antennas was seen in Sections II-B and $\mathrm{C}$ to be $-18 \mathrm{~dB}$ to $-21 \mathrm{~dB}$ for the transmitter antenna and better than $-23 \mathrm{~dB}$ for the receiver antenna (Section II-C). The second order component, which is affected by depolarization at the transmitter and receiver, is considered negligible 


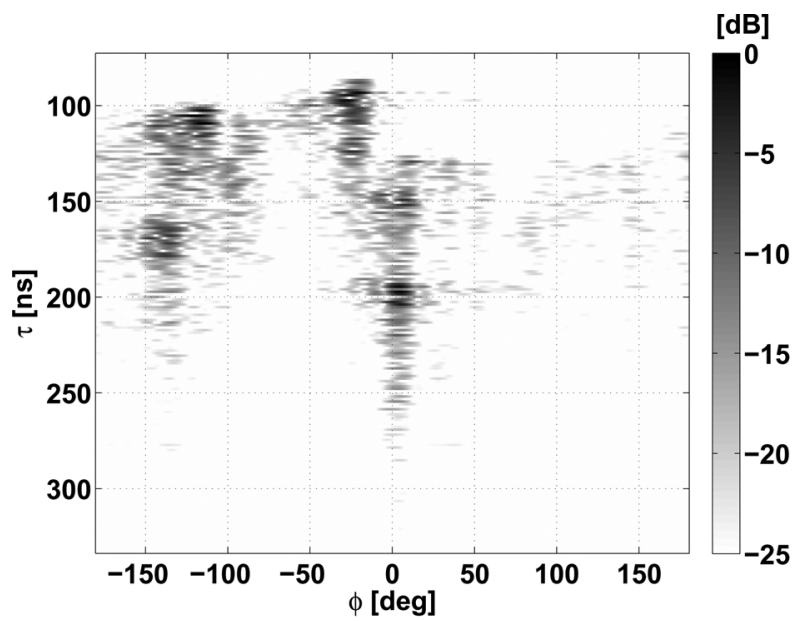

(a) Co-polarized component

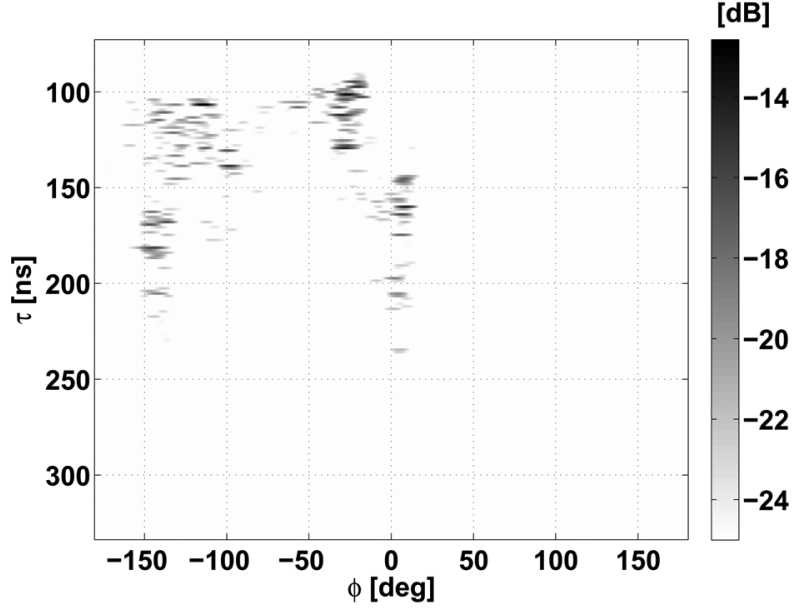

(b) Cross-polarized component

Fig. 11. The MPC power distribution as a function of the ToA $(\tau)$ and the azimuth $\operatorname{AoA}(\phi)$ for scenario B in the horizontal plane $\left(\theta=90^{\circ}\right)$ and for both polarizations.

in comparison. The error sources in the cross-polarized measurement are similar: (a) depolarization at the receiver antenna of the co-polarized component; and (b) depolarization at the transmitter.

In Location A, the error on the co-polarized power measurement is at most $-15 \mathrm{~dB}$, although this applies to only the lower extreme of the frequency band for a narrow range of elevation angles. A more reasonable estimate is $-17 \mathrm{~dB}$. As noted in Section III-B, the peak measured cross-polarized power is $12 \mathrm{~dB}$ lower than the co-polarized power, and hence is more affected by the antenna depolarization effects, giving an error level approximately $5 \mathrm{~dB}$ below the measured cross-polarized component. For the co-polarized power, the error level is approximately $-17 \mathrm{~dB}$.

In Location B, the peak measured cross- and co-polarized power levels are approximately equal, giving error levels of approximately $-17 \mathrm{~dB}$.

The other source of errors is the CLEAN algorithm which was applied to estimate the spatial distribution of co-polarized and cross-polarized MPCs. Simulations using artificially created signals showed that the maximum error expected in the

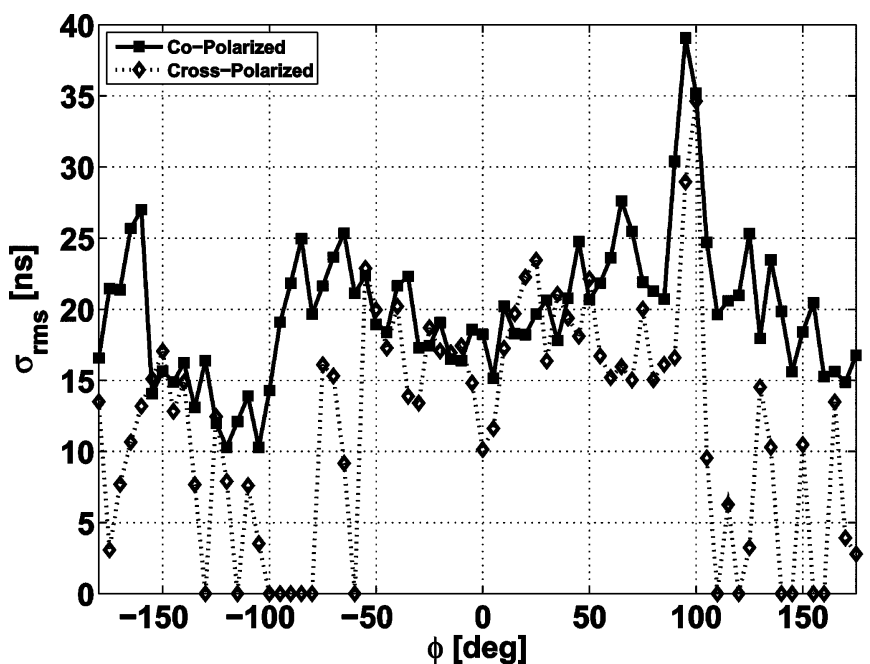

(a) Scenario A

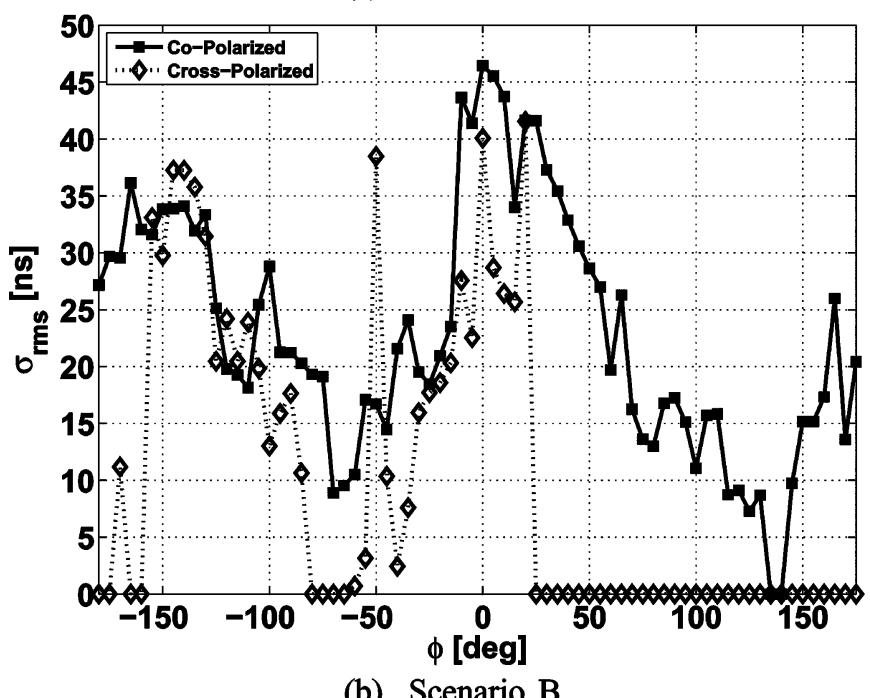

(b) Scenario B

Fig. 12. The rms delay spread for both propagation scenarios in the horizontal plane $\left(\theta=90^{\circ}\right)$ and for both polarizations.

MPC amplitude estimation due to the CLEAN algorithm implementation is approximately $0.4 \mathrm{~dB}$, which is negligible compared to the impact of the antennas.

\section{CONCLUSION}

The power distribution and time dispersion of MPCs have been presented for co- and cross-polarized MPC signals in two indoor scenarios. The joint AoA-ToA power distribution exhibits similar patterns in both indoor scenarios. For instance, the cross-polarized signals exhibit similar power levels for all elevation angles while co-polarized signals appear as a number of localized clusters in and around the horizontal plane $\left(\theta=90^{\circ}\right)$. Furthermore, for some high and low elevation AoAs, the received cross-polarized power, although relatively small, is greater than the co-polarized power.

The MPCs with maximum excess delays, in other words the last MPCs to arrive, for any given AoA are those that have had more interactions with the environment. It was observed that the power of cross-polarized MPCs decays faster than that 
of co-polarized MPCs. This may be explained by the fact that walls act as lossy waveguides favouring the co-polarized signal propagation. Cross-polarized signals originating in the transmitter neighbourhood are attenuated beyond the detection level before arriving at the receiver. Therefore, the detected crosspolarized MPCs are created as a result of the depolarization of co-polarized signals interacting with objects in the receiver neighborhood.

The characteristics of the transmitter and receiver antennas limit the accuracy of the measurements due to depolarization. The impact of the antenna depolarization is greater when the observed cross-polarization is smaller. Of the two locations measured, the one with the lower observed cross-polarization is the one with the larger amount of clutter in the neighbourhood of the transmitter, which militates against accurate ray-tracing analysis. This illustrates the challenges in accurately determining the degree of cross-polarization in some environments.

The rms delay spread estimated for $\theta=90^{\circ}$ indicates a high correspondence between both polarized MPC signals for those azimuth AoAs where the signal power is maximum. Hence, these signals are orthogonally polarized manifestations of the same signal components. The coherent addition of orthogonally polarized signals in systems with adequate MPC resolution can provide an increase in signal-to-noise ratio by collecting this otherwise wasted power. This provides an alternative method to the traditional polarization diversity in multisector antenna systems for indoor communications.

\section{ACKNOWLEDGMENT}

The authors thank the Advanced Antenna Technology research group and C. Squires at the Communications Research Centre for their assistance. The authors are also grateful to Dr. $\mathrm{R}$. Bultitude of CRC for his advice in designing the experiment.

\section{REFERENCES}

[1] W. C.-Y. Lee and Y. S. Yeh, "Polarization diversity systems for mobile radio," IEEE Trans. Commun., vol. 20, pp. 912-923, Oct. 1972.

[2] S. Kozono, T. Tsuruhara, and M. Sakamoto, "Base station polarization diversity reception for mobile radio," IEEE Trans. Veh. Technol., vol. 33, pp. 301-306, Nov. 1984.

[3] D. D. Cox, R. R. Murray, H. W. Arnold, A. W. Norris, and M. F. Wazowicz, "Cross-polarization coupling measured for $800 \mathrm{MHz}$ radio transmission in and around houses and large buildings," IEEE Trans. Antennas Propag., vol. 34, no. 1, pp. 83-87, Jan. 1984.

[4] R. G. Vaughan, "Polarization diversity in mobile communications," IEEE Trans. Veh. Technol., vol. 39, pp. 177-186, Aug. 1990.

[5] M. R. Andrews, P. P. Mitra, and R. deCarvalho, "Tripling the capacity of wireless communications using electromagnetic polarization," $\mathrm{Na}$ ture, vol. 409, pp. 316-318, Jan. 2001.

[6] IEEE 802.11 Standard Group Document, I. Std 802.11g 2003.

[7] IEEE 802.15 Standard Group Document, I. Std 802.15.1-2005.

[8] A. M. Saleh and R. A. Valenzuela, "A statistical model for indoor multipath propagation," IEEE J. Sel. Areas Commun., vol. 5, no. 2, pp. 128-137, Feb. 1987.

[9] Q. H. Spencer, B. D. Jeffs, M. A. Jensen, and A. L. Swindlehurst, "Modeling the statistical time and angle of arrival characteristics of an indoor multipath channel," IEEE Trans. Veh. Technol., vol. 18, pp. 569-571, Mar. 2000.

[10] K. Kalliola, H. Laitinen, L. I. Vaskelainen, and P. Vainikainen, "Realtime 3-D spatial-temporal dual-polarized measurement of wideband radio channel at mobile station," IEEE Trans. Instrum. Meas., vol. 49, pp. 1677-1687, Apr. 2000.
[11] K. Kalliola, K. Sulonen, H. Laitinen, O. Kivekas, J. Krogerus, and P. Vainikainen, "Angular power distribution and mean effective gain of mobile antenna in different propagation environments," IEEE Trans. Veh. Technol., vol. 51, pp. 823-838, Sep. 2002.

[12] X. Yin, B. H. Fleury, P. Jourdan, and A. Stucki, "Polarization estimation of individual propagation paths using the SAGE algorithm," in Proc. IEEE Int. Symp. on Indoor and Mobile Radio Communications (PIMRC), Sep. 2003, pp. 1795-1799.

[13] B. H. Fleury, X. Yin, P. Jourdan, and A. Stucki, "High-resolution channel parameter estimation for communication systems equipped with antenna arrays," in Proc. 13th IFAC Symp. on System Identification (SYSID), Rotterdam, The Netherlands, Aug. 2003.

[14] C.-C. Chong, C.-M. Tan, D. Laurenson, S. McLaughlin, M. Beach, and A. Nix, "A new statistical wideband spatio-temporal channel model for 5-GHz band WLAN systems," IEEE J. Sel. Areas Commun., vol. 21, no. 2, pp. 139-150, Feb. 2003.

[15] N. Czink, X. Y. H.Özcelik, M. Herdin, E. Bonek, and B. H. Fleury, "Cluster characteristics in MIMO indoor propagation environment," IEEE Trans. Wireless Commun., vol. 6, no. 4, pp. 1465-1475, Apr. 2007.

[16] H. L. Bertoni, Radio Propagation for Modern Wireless Systems. Englewood Cliffs, NJ: Prentice-Hall, 1999.

[17] L. Gurrieri, "Spatiotemporal Characterization of Indoor Wireless Channels," M.Sc. thesis, Univ. Manitoba, Elect. Comput. Eng. Dept., Canada, 2006.

[18] IMST GmbH, Team Empire, XCcell EM Simulation Software [Online]. Available: http:/www.empire.de

[19] S. M. Jefferies and J. C. Christou, "Restoration of astronomical images by iterative blind deconvolution," Astronomical J., no. 415, pp. 862-874, Oct. 1993.

[20] J. Tsao and B. D. Steinberg, "Reduction of sidelobe and speckle artifacts in microwave imaging: The CLEAN technique," IEEE Trans. Antennas Propag., vol. 36, no. 4, pp. 543-556, Apr. 1988.

[21] R. J. C. Bultitude, P. Melancon, H. Zaghloul, G. Morrison, and M. Prokki, "The dependence of the indoor multipath channel characteristics on transmit/receive ranges," IEEE Trans. Veh. Technol., vol. 11, pp. 235-245, Feb. 1998.

[22] E. S. Sousa, V. M. Jovanovic, and C. Daigneault, "Delay spread measurements for the digital cellular channel in toronto," IEEE Trans. Veh. Technol., vol. 43, pp. 837-847, Nov. 1994.

[23] M. A. Jensen, Q. H. Spencer, A. L. Swindlehurst, and B. D. Jeffs, "Measurement and modeling of temporal and spatial indoor multipath characteristics," in Proc. IEEE Antennas and Propagation Society Int. Symp., Jul. 1999, pp. 388-391.

[24] M. Landmann, K. Sivasondhivat, J.-I. Takada, I. Ida, and R. Thomä, "Polarization behavior of discrete multipath and diffuse scattering in urban environments at $4.5 \mathrm{GHz}$," EURASIP J. Wireless Commun. Network., no. 1, p. 60, 2007.

[25] L. Gurrieri, C. Squires, S. Noghanian, and T. Willink, "High resolution spatiotemporal characterization of the electric field polarization on indoor environments," in Proc. IEEE Canadian Conf. on Elec. and Comp. Eng. (CCECE/CCGEI'O6), Ottawa, Canada, May 2006, pp. 940-943.

[26] J. Kivinen, X. Zhao, and P. Vainikainen, "Empirical characterization of wideband indoor radio channel at $5.3 \mathrm{GHz}$," IEEE Trans. Antennas Propag., vol. 49, no. 8, pp. 1192-1203, Aug. 2001.

[27] K. Pahlavan and A. H. Levesque, Wireless Information Networks. New York: Wiley, 2005.

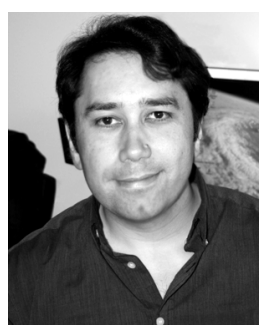

Luis E. Gurrieri received the B.E. degree in electronic engineering specializing in telecommunications from the University of Buenos Aires, Argentina, in 1998 and the M.S. in electrical engineering degree from the University of Manitoba, Winnipeg, MB, Canada, in 2006. He is currently working toward the Ph.D. degree at Queen's University, Kingston, ON, Canada.

From 1998 to 2005, he worked on a number of engineering and $\mathrm{R} \& \mathrm{D}$ projects related to telecommunication systems. Since 2005, he has been working at the Communications Research Centre (CRC), Ottawa, Canada, where he is currently a Research Engineer with the Terrestrial Wireless System group. Since 2007 , his research has focused on cooperative communication systems. 


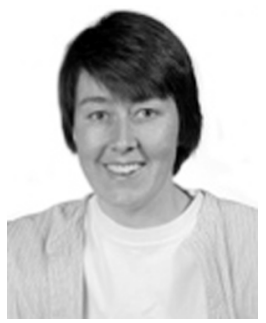

Tricia J. Willink (S'89-M'95-SM'05) received the B.A. and M.A. degrees from Queens' College, Cambridge University, Cambridge, U.K., in 1988 and 1992, respectively, and the Ph.D. degree in electrical engineering from Queen's University, Kingston, ON, Canada, in 1993.

Since 1994, she has been a Research Scientist at the Communications Research Centre, Ottawa, ON, Canada. From 1994 to 2000, she worked in the area of HF communications. Since 2000, her research has focused on signal processing and channel characterization for mobile MIMO communication systems.

Dr. Willink was a co-recipient of the Fred W. Ellersick Prize from the IEEE Communications Society in 2005.

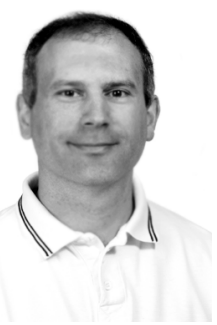

Aldo Petosa (S'89-M'95-SM'02) received the B.Eng., M.Eng., and Ph.D. degrees in electrical engineering from Carleton University, Ottawa, ON, Canada, in 1989, 1991, and 1995, respectively.

From 1990 to 1994, he carried out research at CAL Corporation, Ottawa, on microstrip antennas for cellular and mobile satellite communication applications. In 1995, he joined the Communications Research Centre Canada, Ottawa, Canada, where he is presently the Project Leader for Antenna Design and Development in the Advanced Antenna Technology Lab. He is also an Adjunct Professor with the Department of Electronics, Carleton University. He has published over 100 journal and conference papers and is the author of the Dielectric Resonator Antenna Handbook (Artech House, 2007). His current research interests include microstrip antennas, dielectric lenses, dielectric resonator antennas, and holographic antennas.

Dr. Petosa is the Canadian National Council Chair for International Union of Radio Science (URSI) Commission B.

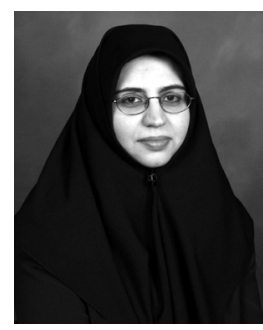

Sima Noghanian (M'03-SM'05) received the B.Sc. degree in electrical engineering from Sharif University of Technology, Tehran, Iran, in 1992 and the M.Sc. and Ph.D. degrees, both in electrical engineering, from the University of Manitoba, Winnipeg, MB, Canada, in 1996 and 2001, respectively.

In 2001, she worked for YottaYotta Corp., Edmonton, Canada, and in 2002 she received a postdoctoral fellowship from the Natural Sciences and Engineering Research Council of Canada (NSERC), which she took at the University of Waterloo. From 2002 to 2003, she was an Assistant Professor in the Electrical Engineering Department, Sharif University of Technology. Since 2003, she has been an Assistant Professor in the Electrical and Computer Engineering Department, University of Manitoba. Her research interest includes antenna design and modeling with applications in wireless communication, wireless channel modeling, ultrawideband antennas and microwave imaging, and application of optimization in antenna design and imaging.

Dr. Noghanian served as the IEEE Winnipeg Waves (joint chapter of Antenna and Propagation/Microwave Theory and Techniques/Vehicular Technology societies) Chair in 2004 and 2005. 